\title{
Laboratory Predictors of Risk for Developing Hepatocellular Carcinoma in Cirrhosis
}

\author{
Wissam Bleibel $^{1 *}$, Saad Saleem ${ }^{2}$, Wael E Saad ${ }^{3}$, Stephen H Caldwell ${ }^{4}$ and Abdullah Al-Osaimi ${ }^{5}$ \\ ${ }^{1}$ Division of Gastroenterology and Hepatology, Department of Medicine, Mercy St. Vincent Medical Centre, Ohio, USA \\ ${ }^{2}$ Internal Medicine Resident, Mercy St. Vincent Medical Center, Ohio, USA \\ ${ }^{3}$ Professor of Radiology, University of Michigan, 1500 E. Medical Center, USA \\ ${ }^{4}$ Professor of Medicine, Division of Gastroenterology and Hepatology, Digestive Health Center, USA \\ ${ }^{5}$ Professor of Medicine, Medical Director of Liver Transplantation, Division of Hepatology, Temple University, USA
}

Received: June 29, 2018; Accepted: July 12, 2018; Published: August 02, 2018

*Corresponding author: Wissam Bleibel, Division of Gastroenterology and Hepatology, Department of Medicine, Mercy St. Vincent Medical Centre, 2213 cherry street, Toledo, Ohio, USA, E-mail: wableibel@yahoo.com

\section{Abstract}

Introduction: Hepatocellular carcinoma (HCC) is a significant cause of mortality and morbidity in patients with cirrhosis. Using laboratory tests to identify patients at high risk for developing HCC may improve outcome and reduce the cost of screening in this patient population.

Methods: In this retrospective study, we included all adult patients evaluated for liver transplantation between 1993 and 2012 at a single university-based transplant center. We used the hospital database and electronic medical records to obtain patients' demographics and laboratory data. ICD-9 code was used to determine patients who developed HCC.

Results: A total of 3284 Patients were included in the study. Patients were predominantly white (86\%), male (56\%), with mixed etiology of cirrhosis (26\% viral, $17 \% \mathrm{EtOH}, 52 \%$ other), had a mean age of 54 years (SD 11.4), MELD of 10 (SD 5.1), albumin 3.7 (SD 0.7), creatinine 1.0 (SD 0.7), INR 1.3 (SD 0.3),bilirubin 1.9 (SD2.8) and platelet count of 149,000 (SD 94,000). After a follow up of 2.7 years (SD 2.7), 5\% developed HCC, $24 \%$ died, and 10\% underwent liver transplantation. In univariate analyses, the following parameters were predictive of HCC risk: age (HR 1.018, 95\% CI 1.004-1.032, p 0.01 ), male gender (HR 3.268, 95\% CI 2.265-4.715, p <0.0001), INR (HR1.896, 95\% CI 1.418-2.534, p <0.0001), albumin (HR 0.453, 95\% CI $0.367-0.561, \mathrm{p}<0.001$ ), platelet count (HR 0.991, 95\% CI 0.989 $0.994, \mathrm{p}<0.0001$ ), bilirubin (HR 1.062, 95\% CI 1.010-1.117, p 0.02) and MELD (HR 1.060, 95\% CI 1.030-1.091, p <0.0001). Creatinine was not predictive. In multivariate analysis, only age, male gender, platelet count, and albumin were predictive of HCC. Patients with platelet count $<100,000$ and albumin $<3$ had the highest annual actuarial risk for HCC of $6.2 \%$.

Conclusion: older males with low platelet count and albumin level are at higher risk for developing HCC. The interval of HCC screening for males with platelet count $<100,000$ and albumin $<3 \mathrm{~g} / \mathrm{dl}$ may need to be shortened. Further prospective studies are warranted to determine the optimal screening interval.

Keywords: Hepatocellular Carcinoma; HCC; Liver; Cirrhosis; Platelet; Albumin; MELD; INR; Bilirubin; Creatinine;

\section{Introduction}

Hepatocellular carcinoma (HCC) is the most common primary liver malignancy worldwide accounting for around 600,000 deaths every year and its incidence has been rising in many countries [1]. In men, it is the fifth most frequently diagnosed cancer worldwide, and the second leading cause of cancerrelated death. In women, it is the seventh most common cancer and the sixth cause of cancer-related death. Despite of significant advances in diagnosis and management, HCC continues to carry a very high case fatality rate $[2,3]$.

The majority of HCC cases (>80\%) are reported in subSaharan Africa and Eastern Asia where the incidence rate exceeds 20 per 100,000 individuals. Southern European countries have lower incidence levels of 10-20 per 100,000, while North America, South America, and Northern Europe have the lowest incidence rate of less than 5 per 100,000 individuals. Over the past few years the incidence of HCC has been decreasing in Japan, Hong Kong, Shanghai, and Singapore while it has been increasing in USA and Canada [4, 5]. The pathogenesis of HCC is complex with the interaction between various factors including intercellular microenvironment, inflammation, oxidative stress, and hypoxia in addition to intracellular and nuclear changes that interact together leading to tumor initiation, progression, and metastasis $[6,7]$. In recent years, it has become clear that innate immunity plays a role in development of HCC through the production of pro-inflammatory cytokines and chemokines [6].

Chronic inflammation and fibrosis associated with chronic liver disease and cirrhosis is the primary trigger of carcinogenesis in the liver [8]. Current guidelines recommend screening highrisk patients with ultrasound (US) examination and serum alpha-fetoprotein (AFP) at 6-month intervals. If a suspicious lesion is identified, further testing with 4-phase CT or dynamic contrast-enhanced MRI should be performed. Liver biopsy can be performed to confirm the diagnosis [9]. Initial screening with US 
alone or in combination with AFP is likely to be the most costeffective strategy [10].

A randomized controlled trial of surveillance with a 6-monthly combination of US and AFP versus no surveillance showed a survival benefit to surveillance. This study was conducted in China and enrolled 18,816 patients. Even though the adherence to surveillance was suboptimal in this study, patients in the surveillance arm had a 37\% reduction in HCC related mortality [11].

In a study by Cabibbo et al., the median survival of patients with untreated HCC was 9.8 (range 6.4-13), 6.1 (range 4.9-7.3), and 3.7 months (range 1.5-6) for Child-Pugh class A, B and C respectively ( $\mathrm{P}<0.05$ for comparison between stages) [12] Detection of HCC in early stages results in survival improvement while HCC detected after the onset of symptoms carries a very poor prognosis (5-year survival of $0-10 \%$ ). In contrast, early diagnosis of HCC offers the potential for cure with studies showing the 5-year survival to exceed 50\% [13]. Determining patients with higher risk for developing HCC may result in improving screening protocols and in lowering the overall cost. Thus, it was the aim of this study to determine demographic and laboratory factors associated with increased risk for developing HCC.

\section{Methods}

The single-center retrospective study that was conducted at our tertiary care transplant center after approval by the internal review board. All adult patients evaluated for liver transplantation at our Center between 1993 and 2012 were included in the study. Paediatric patients and those with a pre-existing diagnosis of HCC were excluded from the study. The clinical data repository at our center was used to gather patients' demographic and laboratory values at the first encounter at the Transplant Center. It included: age, gender, etiology of liver disease, serum bilirubin, serum creatinine, the international normalized ratio (INR), serum albumin, Model for End-Stage Liver Disease score (MELD), and platelet count. The primary endpoint of the study was determined as the development of HCC. Patients who died or underwent liver transplantation were censored in survival analyses. The association between demographics, etiology of liver disease, MELD, MELD components, platelet count, and albumin with risk for developing HCC was investigated. All statistical analyses were performed with SPSS (19.0, Chicago, IL). Categorical variables were compared using chi-square analysis or Fisher exact test where appropriate. Continuous variables were analyzed using the Wilcoxon sign-rank test or independent sample t-test as appropriate. Unadjusted survival was calculated using KaplanMeier estimates. Multivariate survival models were constructed using logistic regression and proportional hazards modelling with an endpoint of death on the waiting list and censoring at the time of liver transplantation using the method of maximum likelihood estimates. Type one error differences at the 0.05 level or less were considered statistically significant. All statistical tests were two-sided.

\section{Results}

A total of 3284 patients were included in the study. Patients were predominantly white, male, had a mean age of 54 years, with mean MELD of 10, albumin 3.7 and platelet count of 149,000. After a mean follow up of 2.7 years, $5 \%$ of the patients developed HCC, $24 \%$ died, and $10 \%$ underwent liver transplantation (Table 1). In univariate analyses, the following parameters were predictive of HCC risk: age (HR 1.018, 95\% CI 1.004-1.032, p 0.01), male gender (HR 3.268, 95\% CI 2.265-4.715, p <0.0001), INR (HR1.896, 95\% CI 1.418-2.534, p <0.0001), albumin (HR 0.453, 95\% CI 0.367$0.561, \mathrm{p}<0.001$ ), platelet count (HR 0.991, 95\% CI 0.989-0.994, $\mathrm{p}<0.0001$ ), Bilirubin (HR 1.062, 95\% CI 1.010-1.117, p 0.02) and MELD (HR 1.060, 95\% CI 1.030-1.091, p <0.0001). Creatinine was not predictive of HCC risk. Older age and male gender were associated with increased risk for HCC. Worse liver function- as indicated by increased bilirubin, INR, and MELD- and decreased albumin, were associated with higher risk for HCC. Patients with lower platelet count had an increased incidence of HCC.

\begin{tabular}{|c|c|c|c|}
\hline \multicolumn{4}{|l|}{ Table 1} \\
\hline & HCC+ & HCC- & $\mathbf{p}$ \\
\hline$\#$ & 165 & 3118 & \\
\hline Age (years) & 55.2 (SD 9.5) & 54 (SD 11.5) & 0.007 \\
\hline Male & $77.6 \%$ & $55.2 \%$ & $<0.0001$ \\
\hline White & $82.4 \%$ & $85.8 \%$ & 0.2 \\
\hline LOS (years) & 2.3 (SD 2.5) & 2.7 (SD 2.7) & 0.07 \\
\hline Platelet & 111.5 (SD 62.6) & 151.1 (SD 95) & $<0.0001$ \\
\hline Creatinine & 0.9 (SD 0.3) & 1.0 (SD 0.7) & 0.02 \\
\hline INR & 1.3 (SD 0.3) & 1.3 (SD 0.4) & 0.05 \\
\hline Bilirubin & 1.9 (SD 1.7) & 1.9 (SD 2.8) & 0.04 \\
\hline Albumin & 3.5 (SD 0.6) & 3.7 (SD 0.7) & 0.02 \\
\hline MELD & 10.2 (SD 4.3) & 10.0 (SD 5.2) & 0.02 \\
\hline $\begin{array}{c}\text { Etiology: } \\
\text { ETOH } \\
\text { Viral } \\
\text { ETOH/Viral } \\
\text { Other }\end{array}$ & $\begin{array}{l}13.3 \% \\
37.6 \% \\
12.7 \% \\
36.4 \%\end{array}$ & $\begin{array}{c}17 \% \\
25.1 \% 4.9 \% \\
53 \%\end{array}$ & $<0.0001$ \\
\hline
\end{tabular}

In multivariate analysis, only age (HR 1.031, 95\% CI 1.0161.046, p <0.0001), male gender (HR 3.013, 95\% CI 2.042-4.444, $\mathrm{p}<0.0001$ ), platelet count (HR 0.993, 95\% CI 0.991-0.996, $\mathrm{p}<0.0001$ ), and albumin (HR 0.511, 95\% CI 0.399-0.654, p $<0.0001$ ) were predictive of risk for HCC. MELD and MELD components failed to reach statistical significance in predicting the risk for developing HCC.

The patients were stratified into two groups: those with a platelet count of $<100,000 / \mu \mathrm{l}$ and those with platelet count $\geq 100,000 / \mu \mathrm{l}$. The two groups were compared to define any differences. Patients with platelet count $<100,000 / \mu$ l were older, white, males with higher MELD scores, and had a higher incidence 
of HCC (Table 2). Cox regression survival analysis showed that patients with platelet count $<100,000 / \mu$ l were at significantly increased risk to develop HCC than those patients with platelet count $\geq 100,000 / \mu \mathrm{l}$ (HR 2.5, 95\% CI 1.82-3.38, p<0.0001). (Figure 1) There was no difference in a time interval to the diagnosis of cancer between the two groups. Similarly, patients were stratified into two groups: those with serum albumin level $<3 \mathrm{~g} / \mathrm{dl}$ vs. patients with an albumin level of $\geq 3$. The lower albumin level was associated with significant increase in risk for HCC (HR 2.4, 95\% CI 1.64-3.49, $\mathrm{p}<0.0001$ ) in Cox regression model. (Figure 2) In multivariate survival analysis including platelet count $<100,000$ and albumin $<3$, both factors were predictive of risk for HCC (HR 2.4, 95\% CI 1.73-3.21, p <0.0001 and HR 2.2, 95\% CI 1.48-3.15, p $<0.0001$ respectively). The patients were further classified into four groups based on platelet count cut-off of $100,000 / \mu \mathrm{l}$ and albumin level cut-off of 3 grams/dl. Group A (platelet $\geq 100,000$ and albumin $\geq 3$ ), B (platelet $<100,000$ and albumin $\geq 3$ ), C (platelet $\geq 100,000$ and albumin $<3$ ), and D (platelet $<100,000$ and albumin $<3$ ). Group A had the highest cancer-free survival while group D had the lowest cancer-free survival. The survival curves of group B and C were similar. (Figure 3) The actuarial risk for developing HCC was $1 \%$ per year for patients with platelet count $\geq 100,000$ and albumin $\geq 3$. The highest risk was $6.2 \%$ per year for those with platelet $<100,000$ and albumin $<3$. The remaining two groups had an intermediate risk of $2.6 \%$ per year.

\begin{tabular}{|c|c|c|c|}
\hline \multicolumn{4}{|l|}{ Table 2} \\
\hline & Plt $<100,000$ & Plt $\geq 100,000$ & $\mathbf{p}$ \\
\hline \# & 1210 & 2073 & \\
\hline Age (years) & 53.8 (SD 10.5) & 54.2 (SD 11.8) & $<0.0001$ \\
\hline Male & $63.6 \%$ & $52.1 \%$ & $<0.0001$ \\
\hline White & $87.3 \%$ & $84.70 \%$ & 0.04 \\
\hline LOS (years) & $2.3(\mathrm{SD} 2.4)$ & 2.9 (SD 2.9) & $<0.0001$ \\
\hline Platelet & 71.6 (SD 17.8) & 195 (SD 90.6) & $<0.0001$ \\
\hline Creatinine & $1.0(\mathrm{SD} 0.8)$ & 1.0 (SD 0.7) & 0.6 \\
\hline INR & $1.4(\mathrm{SD} 0.4)$ & 1.2 (SD 0.3) & $<0.0001$ \\
\hline Bilirubin & 2.3 (SD 2.6) & 1.7 (SD 2.8) & $<0.0001$ \\
\hline Albumin & 3.5 (SD 0.6) & 3.9 (SD 0.7) & $<0.0001$ \\
\hline MELD & 11.3 (SD 5.3) & 9.2 (SD 4.8) & $<0.0001$ \\
\hline $\begin{array}{c}\text { Etiology: } \\
\text { ETOH } \\
\text { Viral } \\
\text { ETOH/Viral } \\
\text { Other }\end{array}$ & $\begin{array}{c}15 \% \\
28.9 \% \\
7.7 \% \\
48.4 \%\end{array}$ & $\begin{array}{c}17.8 \% \\
23.9 \% \\
3.9 \% \\
54.4 \%\end{array}$ & $<0.0001$ \\
\hline HCC & $7.30 \%$ & $3.70 \%$ & $<0.0001$ \\
\hline $\begin{array}{c}\text { Time to HCC } \\
\text { (years) }\end{array}$ & $2.3(\mathrm{SD} 2.4)$ & $2.4(\mathrm{SD} 2.5)$ & 0.7 \\
\hline
\end{tabular}

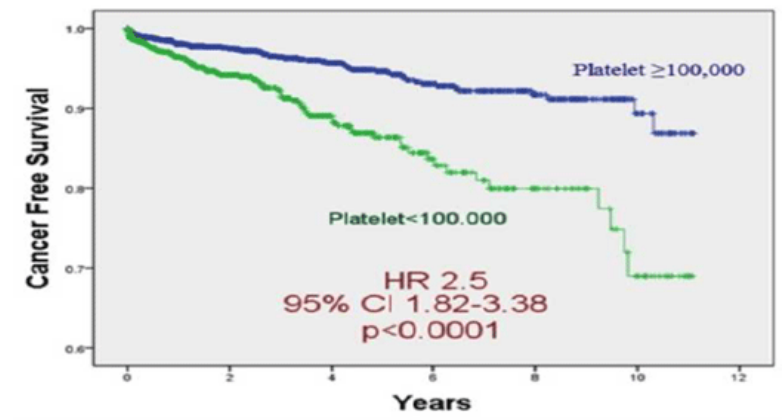

Figure 1: This Kaplan-Meier survival curve demonstrates the higher HCC free rate among patients with serum albumin level $\geq 3$ grams/dl in comparison to those with albumin level $<3$ grams $/$ dl

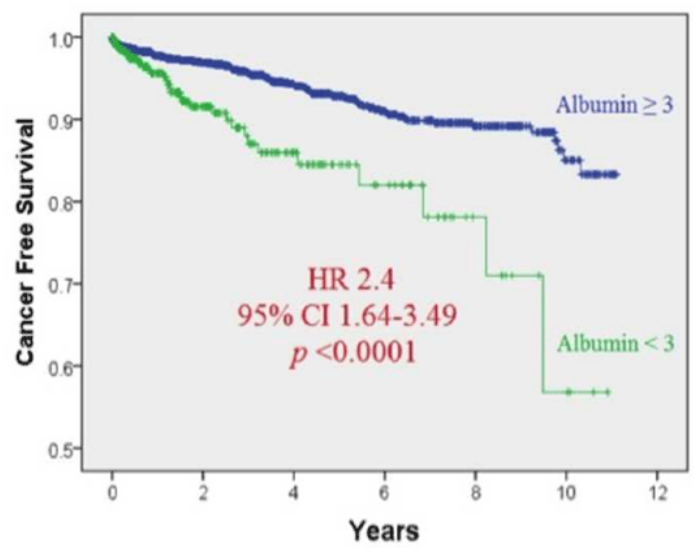

Figure 2: This Kaplan-Meier survival curve demonstrates the higher HCC free rate among patients with serum albumin level $\geq 3$ grams/dl in comparison to those with albumin level $<3$ grams/dl.

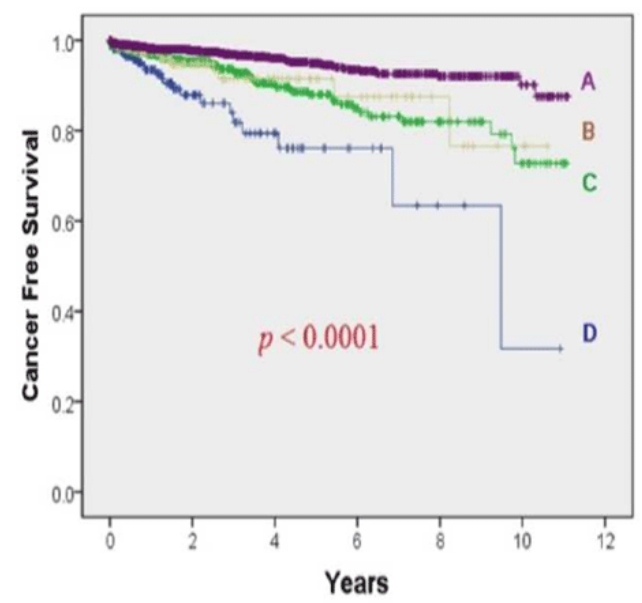

Figure 3: This Kaplan-Meier survival curve compares the HCC free survival of 4 groups of patients based on platelet count cut-off of 100,000/ $\mu \mathrm{l}$ and albumin level cut-off of $3 \mathrm{grams} / \mathrm{dl}$ : A (high platelet and high albumin), B (low platelet and high albumin), C (high platelet and low albumin), and D (low platelet and low albumin). Group A had the highest cancer-free survival while group D had the lowest cancer-free survival. The survival curves of group B and C are very similar. 


\section{Discussion}

Approximately 80 percent of HCC cases are due to underlying chronic viral hepatitis [14]. Epidemiological studies have shown that the majority of cases are diagnosed in older patients with underlying cirrhosis and mean age at presentation of 50 to 60 years [15]. Patients with chronic hepatitis B viral (HBV) infection are at increased risk for developing HCC even in the absence of cirrhosis, yet $70-90 \%$ of HCC cases diagnosed in patients with chronic HBV are in the setting of underlying cirrhosis $[16,17]$. Chronic hepatitis C (HCV) infection has also been shown to be associated with increased risk for developing HCC $[18,19]$. HCV accounts for over one-third of the cases of HCC in the USA (Davila) and around $90 \%$ of these patients have underlying cirrhosis or advanced fibrosis [20]. Over the past decade, the number of HCC cases related to NASH has been increasing [21]. In multivariate analysis, the investigators have identified four factors predictive for development of HCC; those include age, male gender, albumin and low platelet count. Studies have shown that men are at higher risk for developing HCC than women. The cause of this is not fully understood. Possible explanations include the higher incidence of liver diseases in men, higher incidence of environmental exposure to toxins, and the potential effect of androgens [2]. A study conducted on mice revealed a possible cause of gender disparity, chemical carcinogen diethylnitrosamine (DEN) caused a more significant increase in serum interleukin-6 (IL-6) concentration in males as compared to females. In females, estrogen inhibited the increases of IL-6 in response to DEN [22]. Our study showed that older males with low platelet count and low serum albumin are at higher risk for developing HCC. In a study by Chang et al., thrombocytopenia, older age, male gender, higher stage of hepatic fibrosis, genotype 1 , and failure to eradicate HCV were shown to be associated with increased risk for developing HCC after interferon-based treatment [23]. In another study by Velazquez et al., a total of 463 patients aged 40 to 65 years with liver cirrhosis in Child-Pugh class A or B were evaluated to determine predictive factors of HCC. In their multivariate analysis, the independent predictive factors for the development of HCC included: age $\geq 55$ years, anti-HCV positivity, prothrombin activity $\leq 75 \%$, and platelet count $<75,000 / \mathrm{mm}$ [24]. Thrombocytopenia is a common complication of cirrhosis affecting up to $76 \%$ of this patient Population. Several studies have shown an association between low platelet count and development of HCC $[25,26]$. The etiology of thrombocytopenia associated with cirrhosis is multifactorial [27]. It has been for many years considered to be simply a result of portal hypertension and splenomegaly. Recent studies have suggested a direct role of cirrhosis and loss of liver mass, due to parenchymal extinction caused by microthrombosis, in lowering the production of thrombocytes via decreased levels of circulating thrombopoietin (TPO) [28, 29].Thrombocytopenia can be seen in cirrhosis patients with normal spleen size [30]. Also, the reduction of portal pressure using portosystemic shunting does not frequently correct thrombocytopenia [31, 32]. A study by El-Sayed et al. confirmed that lower platelet counts and TPO levels correlated with increased severity of liver disease graded by Child-Turcotte-Pugh (CTP) scoring system [33]. Furthermore,
Koruk et al. demonstrated a similar correlation between the severity of liver disease grade by CTP classification and serum levels of TPO [34]. In a recent study, we have shown that cirrhotic patients with lower platelet counts have increased mortality rate while awaiting liver transplantation [35]. These data indicate that the severity of thrombocytopenia correlates with the severity of liver fibrosis. In a study by Sato et al., low albumin level has been shown to be associated with increased risk for developing HCC after eradication of HCV infection [36]. Similarly, low albumin level was associated with increased risk for HCC in cirrhosis patients with no HBV or HCV infection [37]. A study conducted in Switzerland suggested that albumin suppresses cell proliferation, hence suppressing the proliferation of hepatocellular carcinoma [38].

Despite the advances in diagnosis and management of HCC a retrospective study from Germany compared the outcome of 385 cases of HCC diagnosed between 1998 and 2003 and 681 cases diagnosed between 2004 and 2009 and found no difference in overall survival between the two time periods. The authors attributed this to the more advanced stage of HCC and increasing age at the time of diagnosis in the second period [39]. Other studies have shown an increased number of HCC cases and improved outcome with current treatment protocol [40]. Thus, diagnosis of HCC at earlier stages via screening protocols may potentially reduce morbidity and mortality.

Based on the findings of our study older cirrhotic patients with lower platelet count and serum albumin level might be at increased risk for developing HCC than other patients. This subgroup of patients may benefit from more frequent interval screening examinations. A cut-off platelet count of $100,000 / \mu \mathrm{l}$ showed a 2.5-fold increase in risk for HCC in patients with lower platelet count. A similar increase in HCC risk was noted in patients with albumin level $<3$ grams/dl in comparison to patients with higher albumin level. The highest risk (annual actuarial risk of $6.2 \%$ ) was seen in patients with a combination of platelet count $<100,000$ and albumin $<3$. This subgroup of patients (especially males) may benefit from more intensive screening protocol (every 4 months US and AFP). The lowest annual actuarial risk (1\%) was seen in patients with platelet count $\geq 100,000$ and albumin $\geq 3$. This group may be screened once per year. The remaining groups with either high platelet count or high albumin count may be screened every six months. Large-scale studies are required to evaluate these findings further. Despite the advances in diagnosis and management of HCC a retrospective study from Germany compared the outcome of 385 cases of HCC diagnosed between 1998 and 2003 and 681 cases diagnosed between 2004 and 2009 and found no difference in overall survival between the two time periods. The authors attributed this to the more advanced stage of HCC and increasing the age at the time of diagnosis in the second period [39]. Other studies have shown an increased number of HCC cases and improved outcome with current treatment protocols [40]. Thus, diagnosis of HCC at earlier stages via screening protocols may potentially reduce morbidity and mortality. 


\section{Conclusion}

Older cirrhotic males with low platelet count and low serum albumin are at higher risk for developing HCC. The interval of HCC screening may warrant further prospective studies with shortened (every four months) for males with a platelet count of less than 100,000/ $\mu$ land albumin of fewer than 3 grams/dl. Patients with high platelet count and high albumin level may be screened less intensely.

\section{Acknowledgment}

- The authors would like to thank Kenneth Scully, University of Virginia

- Clinical Data Repository, for assisting in data acquisition.

\section{References}

1. Ferenci P, Fried M, Labrecque D, Bruix J, Sherman M, Omata M,et al. World Gastroenterology Organisation Guideline. Hepatocellular carcinoma (HCC): a global perspective. J Gastrointestin Liver Dis. 2010;19(3):311-317.

2. Jemal A, Bray F, Center MM, Ferlay J, Ward E, Forman D. Global cancer statistics. CA Cancer J Clin. 2011;61(2):69-90. doi: 10.3322/ caac. 20107

3. Altekruse SF, McGlynn KA, Reichman ME. Hepatocellular carcinoma incidence, mortality, and survival trends in the United States from 1975 to 2005. J Clin Oncol. 2009;27(9):1485-1491. doi: 10.1200/ JC0.2008.20.7753

4. El-Serag HB. Epidemiology of viral hepatitis and hepatocellular carcinoma. Gastroenterology. 2012;142(6):1264-1273. doi: 10.1053/j.gastro.2011.12.061

5. Mittal S, El-Serag HB. Epidemiology of hepatocellular carcinoma: consider the population. J Clin Gastroenterol. 2013;47: S2-6. doi: 10.1097/MCG.0b013e3182872f29

6. Aravalli RN. Role of innate immunity in the development of hepatocellular carcinoma. World J Gastroenterol. 2013;19(43):75007514. doi: 10.3748/wjg.v19.i43.7500

7. Aravalli RN, Cressman EN, Steer CJ. Cellular and molecular mechanisms of hepatocellular carcinoma: an update. Arch Toxicol. 2013;87(2):227-247. doi: 10.1007/s00204-012-0931-2

8. Severi T, van Malenstein H, Verslype C, van Pelt JF. Tumor initiation and progression in hepatocellular carcinoma: risk factors, classification, and therapeutic targets. Acta Pharmacol Sin. 2010;31(11):1409-1420. doi: 10.1038/aps. 2010

9. de Lope CR, Tremosini S, Forner A, Reig M, Bruix J. Management of HCC. J Hepatol. 2012;56(1):S75-87. doi: 10.1016/S01688278(12)60009-9

10.Ruggeri M. Hepatocellular carcinoma: cost-effectiveness of screening. A systematic review. Risk Manag Healthc Policy. 2012;5:49-54. doi: 10.2147/RMHP.S18677

11.Zhang BH, Yang BH, Tang ZY. Randomized controlled trial of screening for hepatocellular carcinoma. J Cancer Res Clin Oncol. 2004;130(7):417-422. DOI: 10.1007/s00432-004-0552-0
12. Cabibbo G, Maida M, Genco C, Pietro Parisi, Marco Peralta, Michela Antonucci, et al. Natural history of untreatable hepatocellular carcinoma: A retrospective cohort study. World J Hepatol. 2012;4(9):256-261. doi: 10.4254/wjh.v4.i9.256

13.Yang JD, Harmsen WS, Slettedahl SW, Chaiteerakij R, Enders FT, Therneau TM, et al. Factors that affect risk for hepatocellular carcinoma and effects of surveillance. Clin Gastroenterol Hepatol. 2011;9(7):617-623. doi: 10.1016/j.cgh.2011.03.027

14.Perz JF, Armstrong GL, Farrington LA, Hutin YJ, Bell BP. The contributions of hepatitis $B$ virus and hepatitis $C$ virus infections to cirrhosis and primary liver cancer worldwide. J Hepatol. 2006;45(4):529-538. DOI: 10.1016/j.jhep.2006.05.013

15. Tsukuma H, Hiyama T, Tanaka S, Nakao M, Yabuuchi T, Kitamura T, et al. Risk factors for hepatocellular carcinoma among patients with chronic liver disease. N Engl J Med. 1993;328(25):1797-1801.

16.Sherman M, Peltekian KM, Lee C. Screening for hepatocellular carcinoma in chronic carriers of hepatitis B virus: incidence and prevalence of hepatocellular carcinoma in a North American urban population. Hepatology. 1995;22(2):432-8.

17. Ming-Whei Yu, Chien-Jen Chen. Hepatitis B and C viruses in the development of hepatocellular carcinoma. Crit Rev Oncol Hematol. 1994;17(2):71-91.

18. Bruix J, Barrera JM, Calvet X, Ercilla G, Costa J, Sanchez-Tapias JM, et al. Prevalence of antibodies to hepatitis $C$ virus in Spanish patients with hepatocellular carcinoma and hepatic cirrhosis. Lancet. 1989;2(8670):1004-1006.

19. Colombo M, Kuo G, Choo QL, Donato MF, Del Ninno E, Tommasini MA, et al. Prevalence of antibodies to hepatitis $\mathrm{C}$ virus in Italian patients with hepatocellular carcinoma. Lancet. 1989;2(8670):1006-1008.

20.0mland LH, Jepsen P, Krarup H, Christensen PB, Weis N, Nielsen $\mathrm{L}$, et al. Liver cancer and non-Hodgkin lymphoma in hepatitis $\mathrm{C}$ virus-infected patients: results from the DANVIR cohort study. Int J Cancer. 2012 ;130(10):2310-2317. doi: 10.1002/ijc.26283

21. Fenoglio L, Serraino C, Castagna E, Cardellicchio A, Pomero F, Grosso $\mathrm{M}$, et al. Epidemiology, clinical-treatment patterns and outcome in 256 hepatocellular carcinoma cases. World J Gastroenterol. 2013;19(21):3207-3216. doi: 10.3748/wjg.v19.i21.3207

22. Naugler WE, Sakurai T, Kim S, Maeda S, Kim K, Elsharkawy AM, et al. Gender disparity in liver cancer due to sex differences in MyD88dependent IL-6 production. Science. 2007;317(5834):121-124.

23. Chang KC, Wu YY, Hung CH, Lu SN, Lee CM, Chiu KW, et al. Clinicalguide risk prediction of hepatocellular carcinoma development in chronic hepatitis $\mathrm{C}$ patients after interferon-based therapy. Br J Cancer. 2013 ;109(9):2481-2488. doi: 10.1038/bjc.2013.564

24.Velázquez RF, Rodríguez M, Navascués CA, Linares A, Pérez $\mathrm{R}$, Sotorríos NG, et al. Prospective analysis of risk factors for hepatocellular carcinoma in patients with liver cirrhosis. Hepatology. 2003;37(3):520-527.

25.Lu SN, Wang JH, Liu SL, Hung CH, Chen CH, Tung HD, et al. Thrombocytopenia as a surrogate for cirrhosis and a marker for the identification of patients at high-risk for hepatocellular carcinoma. Cancer. 2006;107(9):2212-2222. 
26. Kubo S, Tanaka H, Shuto T, Takemura S, Yamamoto T, Uenishi T, et al. Correlation between low platelet count and multicentricity of hepatocellular carcinoma in patients with chronic hepatitis C. Hepatol Res. 2004;30(4):221-225.

27. Giannini EG1, Savarino V. Thrombocytopenia in liver disease. Curr Opin Hematol. 2008;15(5):473-480. doi: 10.1097/ MOH.0b013e32830a9746

28. Anstee QM, Dhar A, Thursz MR. The role of hypercoagulability in liver fibrogenesis. Clin Res Hepatol Gastroenterol. 2011;35(89):526-533. doi: 10.1016/j.clinre.2011.03.011

29. Anstee QM, Wright M, Goldin R, Thursz MR. Parenchymal extinction: coagulation and hepatic fibrogenesis. Clin Liver Dis. 2009;13(1):117126. doi: 10.1016/j.cld.2008.09.013

30.Shah SH, Hayes PC, Allan PL, Nicoll J, Finlayson ND. Measurement of spleen size and its relation to hypersplenism and portal hemodynamics in portal hypertension due to hepatic cirrhosis. Am J Gastroenterol. 1996;91(12):2580-2583.

31. Jabbour N1, Zajko A, Orons P, Irish W, Fung JJ, Selby RR. Does transjugular intrahepatic portosystemic shunt (TIPS) resolve thrombocytopenia associated with cirrhosis?. Dig Dis Sci. 1998;43(11):2459-2462.

32. Sanyal AJ, Freedman AM, Purdum PP, Shiffman ML, Luketic VA. The hematologic consequences of transjugular intrahepatic portosystemic shunts. Hepatology Hepatology. 1996;23(1):32-39.

33. El-Sayed R, El-Ela MA, El-Raziky MS, Helmy H, El-Ghaffar AA, El-Karaksy H. Relation of serum levels of thrombopoietin to thrombocytopenia in extrahepatic portal vein obstruction versus cirrhotic children. J Pediatr Hematol Oncol. 2011;33(7):267-270. doi: 10.1097/MPH.0b013e31821d80be
34.Koruk M, Onuk MD, Akçay F, Savas MC. Serum thrombopoietin levels in patients with chronic hepatitis and liver cirrhosis, and its relationship with circulating thrombocyte counts. Hepato gastroenterology. 2002;49(48):1645-1648.

35. Bleibel W, Caldwell SH, Curry MP, Northup PG. Peripheral platelet count correlates with liver atrophy and predicts long-term mortality on the liver transplant waiting list. Transpl Int. 2013;26(4):435442. doi: 10.1111/tri.12064

36. Sato A, Sata M, Ikeda K, Kumada T, Izumi N, Asahina Y, et al. Clinical Characteristics of Patients who developed Hepatocellular Carcinoma after Hepatitis C Virus Eradication with Interferon Therapy: Current Status in Japan. Intern Med. 2013;52(24):27012706.

37. Yamada S1, Kawaguchi A, Kawaguchi T, Fukushima N, Kuromatsu $\mathrm{R}$, Sumie $\mathrm{S}$, et al. Serum albumin level is a notable profiling factor for non-B, non-C hepatitis virus-related hepatocellular carcinoma: A data-mining analysis. Hepatol Res. 2014;44(8):837-845. doi: 10.1111/hepr.12192

38. Nojiri S, Joh T. Albumin suppresses human hepatocellular carcinoma proliferation and the cell cycle. Int J Mol Sci. 2014;15(3):5163-5174. doi: 10.3390/ijms15035163

39. Weinmann A, Koch S, Niederle IM, Schulze-Bergkamen H, König J, Hoppe-Lotichius M, et al. Trends in Epidemiology, Treatment, and Survival of Hepatocellular Carcinoma Patients Between 1998 and 2009: An Analysis of 1066 Cases of a German HCC Registry. J Clin Gastroenterol. 2014;48(3):279-289. doi: 10.1097/ MCG.0b013e3182a8a793

40.Erhardt A1, Zhu E, Blondin D, et al. Increasing number and improved survival of patients with hepatocellular carcinoma from 1988 to 2007: data of a German university clinic. Z Gastroenterol 2011:49(6):720-727. doi: 10.1055/s-0029-1246060 